\title{
Expression of phospholipase D2 in human colorectal carcinoma
}

\author{
MASARU SAITO, MANABU IWADATE, MASASHI HIGASHIMOTO, \\ KOICHIRO ONO, YUJI TAKEBAYASHI and SEIICHI TAKENOSHITA \\ Department of Surgery II, Fukushima Medical University School of Medicine, Fukushima, Japan
}

Received May 21, 2007; Accepted July 30, 2007

\begin{abstract}
Phospholipase D (PLD) catalyzes the hydrolysis of phosphatidylcholine (PC) to generate phosphatidic acid (PA) and choline. PA acts as a second messenger in cell proliferation; therefore PLD is believed to play an important role in carcinogenesis. PLD activity has been reported to be elevated in human breast, gastric, renal cell and colorectal carcinomas, compared with adjacent non-neoplastic tissues. The activity of PLD was also correlated with nuclear grade in breast cancer, tumor size in gastric carcinoma, and nodal involvement and deeper invasion in colorectal carcinoma. However, the number of cases in each study was small. The aim of this study was to investigate the expression level of PLD2 and its association with clinicopathological features in human colorectal carcinoma. Ninety-seven colorectal carcinomas were obtained from surgery. Expression level of PLD2 was assessed by real-time PCR. The prognostic relevance of PLD2 expression level in patients with colorectal carcinoma was also analyzed by the survival analysis of mortality follow-up data covering the period 2000-2004. PLD expression level was varied from tumor to tumor. Expression level of PLD was significantly correlated with tumor size $(\mathrm{P}<0.05)$; it was independent of lymph node metastasis, extent of invasion, pathological classification, distant metastasis and Dukes' stage. PLD expression level was also significantly correlated with survival of patients with colorectal carcinoma $(\mathrm{P}<0.05)$. These findings suggested that PLD2 plays an important role in progression of colorectal carcinoma and that PLD2 could be a target for therapy in colorectal carcinoma.
\end{abstract}

Correspondence to: Dr Yuji Takebayashi, Department of Surgery II, Fukushima Medical University School of Medicine, Hikarigaoka-1, Fukushima 960-1295, Japan

E-mail: tyuji@fmu.ac.jp

Abbreviations: PLD2, phospholipase D2; PC, phosphatidylcholine; PA, phosphatidic acid; G3PDH, glyceraldehydes-3-phosphatedehydrogenase

Key words: phospholipase D2, colorectal carcinoma, real-time PCR

\section{Introduction}

Phospholipase D (PLD) catalyzes the hydrolysis of phosphatidylcholine (PC) to generate phosphatidic acid (PA) and choline. PLD has been implicated in membrane trafficking, cytoskeletal reorganization, receptor endocytosis, exocytosis and cell migration (1). A role for PLD in cell proliferation is indicated from reports showing that PLD activity is elevated in response to platelet-derived growth factor (PDGF) (2), fibroblast growth factor $(3,4)$, epidermal growth factor (EGF) (5), insulin (6), insulin-like growth factor 1 (7), growth hormone (8) and sphingosine 1-phosphate (9). PLD activity is also elevated in cells transformed by a variety of transforming oncogenes including $\mathrm{v}$-Src (10), v-Ras (11), v-Fps (12) and V-Raf (13). Therefore PLD is believed to play an important role in carcinogenesis. In addition, PLD activity was increased in multidrug resistant (MDR) breast cancer cells (14) and PLD conferred rapamycin resistance in human breast cancer cells (15). It has been reported that PLD activity was elevated in human breast carcinoma $(16,17)$, gastric carcinoma (18), renal cancer (19) and colorectal carcinoma (20). Moreover, the activity was correlated with nuclear grade in breast cancer (16), tumor size in gastric carcinoma (18), nodal involvement and deeper invasion in colorectal carcinoma (20). However, the clinicopathologic significance still remains unclear. Since, a large number of clinical analysis for PLD2 expression level has not been reported yet, the aim of this study is to investigate the relationship between PLD2 expression level and clinicopathologic relevance and its prognostic value in human colorectal carcinoma.

\section{Materials and methods}

Patients and tumor samples. We examined 97 patients (61 males and 36 females) whose tumors were completely removed surgically in Department of Surgery of Fukushima Medical University Hospital, Japan, between October 2000 through April 2004. The patients in this study had no other cancers. Tumor specimens were collected after obtaining informed consent in accordance with the institutional guidelines. Samples were immediately stored at $-80^{\circ} \mathrm{C}$ and used for RT-PCR of PLD2. The clinicopathologic characteristics of 97 patients with colorectal carcinomas investigated in this study are summarized in Table I. The average age at surgery was 66 years (range 43-84 years). Patients with Dukes' C 
Table I. Relationship of PLD2 expression detected by real-time PCR and clinicopathological variables in patients with colorectal carcinoma $(n=97)$.

\begin{tabular}{lccc}
\hline Variables & $\begin{array}{c}\text { PLD2 } \\
\text { high (\%) }\end{array}$ & $\begin{array}{c}\text { PLD2 } \\
\text { low }(\%)\end{array}$ & $\begin{array}{c}\text { Signifi- } \\
\text { cance }\end{array}$ \\
\hline
\end{tabular}

No. of patients

Gender

NS

Male

$33(54.1) \quad 28(45.9)$

Female

$15(41.7) \quad 21(58.3)$

Tumor location

rt.

$18(563) \quad 14(43.8)$

It.

$30(46.2) \quad 35(53.8)$

Histopathologic type

Well differentiated

$31(51.7) \quad 29(48.3)$

Moderately differentiated

$15(50.0) \quad 15(50.0)$

Poorly differentiated

Mucinous

$2(50.0) \quad 2 \quad(50.0)$

$\begin{array}{lll}0 & (0.0) \quad 2(100.0)\end{array}$

Squamous

$\begin{array}{lll}0 & (0.0) \quad 1(100.0)\end{array}$

Depth of invasion

Mucosa/submucosa

Muscle layer

Subserosa/serosa

Invasion to adjacent

Lymph node metastasis

$\begin{array}{lll}\text { Absent } & 24(45.3) & 29(54.7) \\ \text { Present } & 24(54.5) & 20(45.5)\end{array}$

Distant metastasis

$\begin{array}{lrr}\text { Absent } & 38(47.5) & 42(52.5) \\ \text { Present } & 10(58.8) & 7(41.2)\end{array}$

Dukes' stage

$\begin{array}{lrr}\text { A } & 6(50.0) & 6(50.0) \\ \text { B } & 14(41.2) & 20(58.8) \\ \text { C } & 28(54.9) & 23(45.1)\end{array}$

P-values were obtained from the $\chi^{2}$ test (two-sided). NS, not significant.

stage disease received postoperative therapy; however there was no difference in outcome among the various treatment modalities. Follow-up for the patients included in the survival analysis was updated in December 2005 (median follow-up was 18 months range 1-52 months). Fourteen patients had died of colorectal carcinoma, 83 were alive. Tumors were classified histopathologically according to the Japanese Classification of colorectal carcinoma. All routine sections were carefully investigated to identify venous or lymphatic invasion. Histopathologic diagnosis was made routinely at
Department of Pathology of Fukushima Medical University Hospital. The largest diameter of the tumor was defined as the tumor size. The extent of tumor invasion/metastasis was based on the Astler and Coller modification of Dukes' classification system. Dukes' A (A1+A2) cases were those in which the growth was confined to the submucosa of the colorectal wall. In Dukes' B (B1+B2) cases, the growth spread by direct continuity into the extracolorectal tissues, but the lymph nodes were free from metastasis. Dukes' C $(\mathrm{C} 1+\mathrm{C} 2)$ cases were those in which lymph node metastasis was found.

RT-PCR analysis. Frozen block of the carcinoma tissues were stained by hematoxylin and eosin to examine the presence and proportion of carcinoma cells. The total RNA was isolated from the sample containing more than $80 \%$ of carcinoma cells with Trizol reagent (Invitrogen, Life Technologies). However, if there were no or a few carcinoma cells, the sample was excluded from this study. cDNA was synthesized from $3 \mu \mathrm{g}$ total RNA using random hexadeoxynucleotide primers (Invitrogen, Life Technologies, Gaithersburg, MD) in a 9.5- $\mu 1$ solution containing reverse transcriptase (Invitrogen, Life Technologies). cDNA was diluted 4-fold in water and stored at $-20^{\circ} \mathrm{C}$ until use. PCR was performed with cDNA derived from $3 \mu \mathrm{g}$ RNA in a total volume of $25 \mu 1$ containing cDNA, dGTP, dATP and dCTP at a concentration of $200 \mu \mathrm{M}, 4 \mu \mathrm{M}$ of each primer and $0.25 \mathrm{U}$ of ExTaq polymerase (Takara Shuzo, Otsu, Shiga). The PCR primer sequences of hPLD2 and glyceroldehyde-3phosphate-dehydrogenase (G3PDH) (used as an internal control) were as follows: hPLD2, forward primer nucleotides 5'-CGCATCCCCATTCCCACTAG 3' and reverse primer nucleotides 5'-AGCAATTCAAGCCT GGT-3' corresponding to 358-bp (residues 211-568); G3PDH, 5'-CCCCTGGCC AAGGTCATCCATGACAACTTT-3' and reverse primer nucleotides 5'-GGCCATGAGGTCCACCACCCTGTTGCT GTA-3' corresponding to 513-bp (residues 515-1027). The PCR amplification was performed for 35 cycles with a $1 \mathrm{~min}$ denaturation step at $94^{\circ} \mathrm{C}$, a 1 -min annealing step at $55^{\circ} \mathrm{C}$, and a 1 -min extension step at $72^{\circ} \mathrm{C}$, followed by a $10-$ min extension step at $72^{\circ} \mathrm{C}$. PCR products were determined in $2 \%$ agarose gels, and visualized by ethidium bromide staining.

Quantitative real-time PCR. For quantification, cDNA was used as template in $\mathrm{IQ}^{\mathrm{TM}} \mathrm{SYBR}^{\mathrm{R}}$ Green Supermix (Bio-Rad, Hercules, CA). The constituents of each PCR (15 $\mu 1)$ were $1 \mu 1 \mathrm{cDNA}, 0.8 \mu 1$ primer $(15 \mathrm{pmol} / 1$ each $), 7.5 \mu 1 \mathrm{IQ}^{\mathrm{TM}}$ $\mathrm{SYBR}^{\mathrm{R}}$ Green Supermix (Bio-Rad). Product amplification was done up to 50 PCR cycles, after uracil removal (2 min at $\left.50^{\circ} \mathrm{C}\right)$ and polymerase activation $\left(15 \mathrm{~min}\right.$ at $\left.95^{\circ} \mathrm{C}\right)$. Each 3 -step PCR cycle comprised denaturing $\left(30 \mathrm{sec}\right.$ at $\left.94^{\circ} \mathrm{C}\right)$, annealing $\left(30 \mathrm{sec}\right.$ at $\left.55^{\circ} \mathrm{C}\right)$ and extending $\left(1 \mathrm{~min}\right.$ at $\left.72^{\circ} \mathrm{C}\right)$.

Evaluation of PCR product detected by quantitative real-time $P C R$. PCR products of each gene examined were synthesized using RNA from placenta described as above. PCR products were electrophoresed on $2 \%$ agarose gels and isolated using RECOCHIP (Takara Shuzo, Otsu, Shiga). After ethanol precipitation, PCR products were diluted with distilled water and quantified with SmartSpec ${ }^{\mathrm{TM}} 3000$ (Bio-Rad, Hercules, 

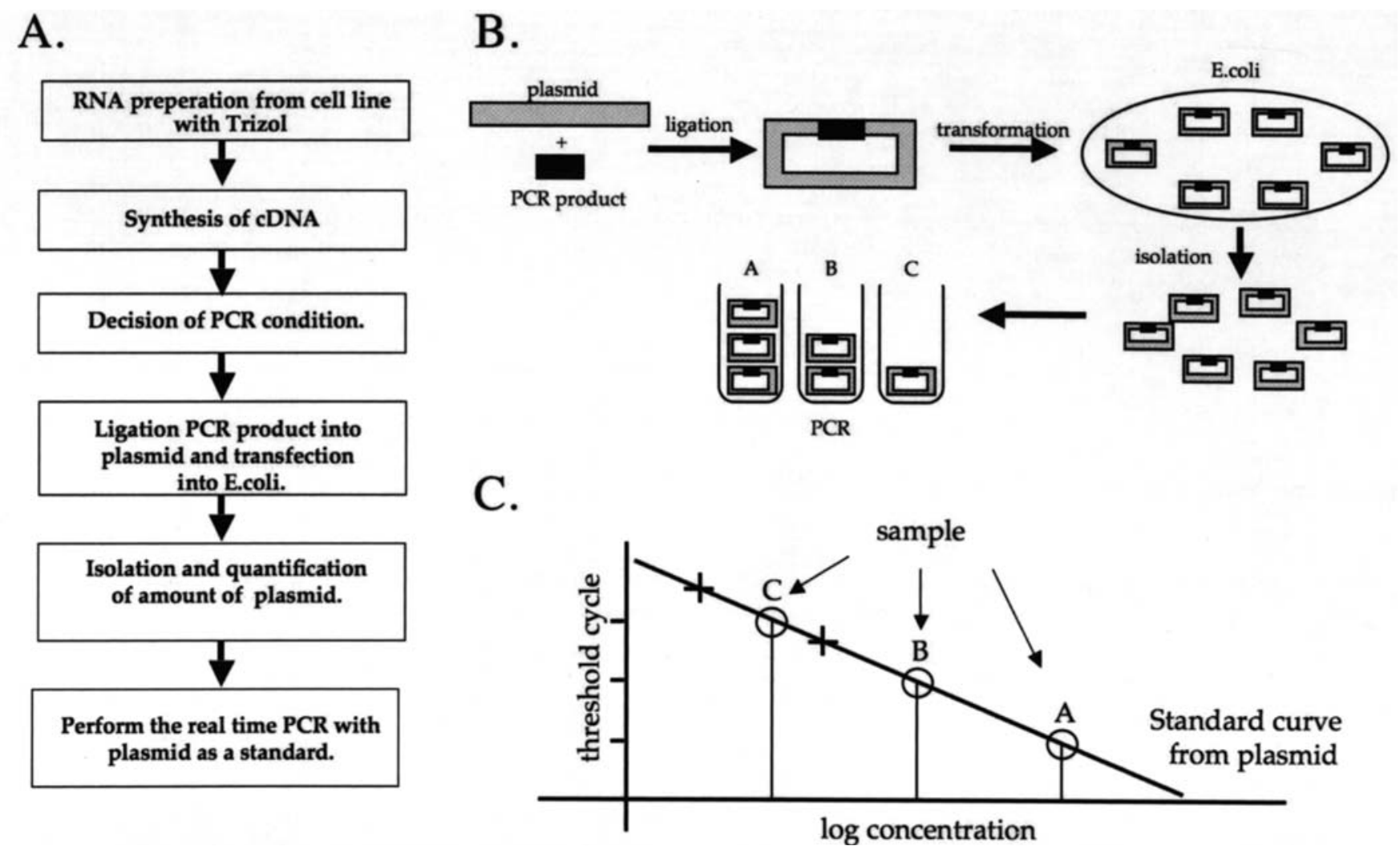

Figure 1. Evaluation of PCR product detected by real-time PCR. (A) Procedure of real-time PCR. (B) Scheme of real-time PCR. (C) Evaluation of PCR product using plasmid with a target of PCR product.

CA). PCR products solution was diluted to $0.3 \mathrm{pmol} / 1,2 \mu 1$ of which was ligated using TOPO ${ }^{\circledR}$ TA cloning kit Dual Promoter (with pCR II-TOPO) (Invitrogen Corp., Carlsbad, CA) by the manufacturer's protocol. Plasmid was transformed into competent cells using TOPO ${ }^{\circledR}$ TA cloning kit Dual Promoter (with pCR II-TOPO). Each transformed plasmid was isolated using Plasmid Mini Kit (Qiagen, Hilden, Germany) by the manufacturer's protocol, and the purified plasmids were diluted at a concentration of $1,0.1$ and $0.01 \mathrm{ng} / \mu 1$ for the standard curve of quantitative real-time PCR, respectively. Finally, the amount of PCR products detected by real-time PCR was described as relative concentration of PLD2 divided by that of G3PDH as a reference of each amount of PCR products using plasmid cosntaining each PCR product (Fig. 1).

Statistical analysis. Demographic and clinicopathologic characteristics were compared with the use of the $\chi^{2}$, Student's t-tests and Spearman's test. The relationship between tumor size and each gene expression was also analyzed by box plot analysis. All P-values presented were two-sided.

\section{Results}

Relationship between clinicopathologic findings and PLD2 expression. PLD2 expression in human colorectal carcinoma was determined by RT-PCR (Fig. 2A). The classification of PLD2 expression level was classified into PLD2 high and PLD2 low by the median value detected by real-time PCR. PLD2 expression level was varied from tumor to tumor (Fig. 2B). Table I summarizes the relationship between
A.

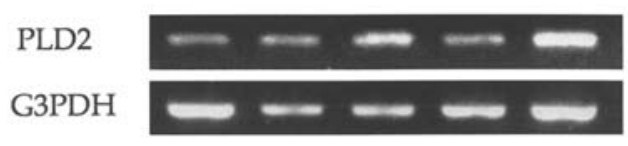

B.

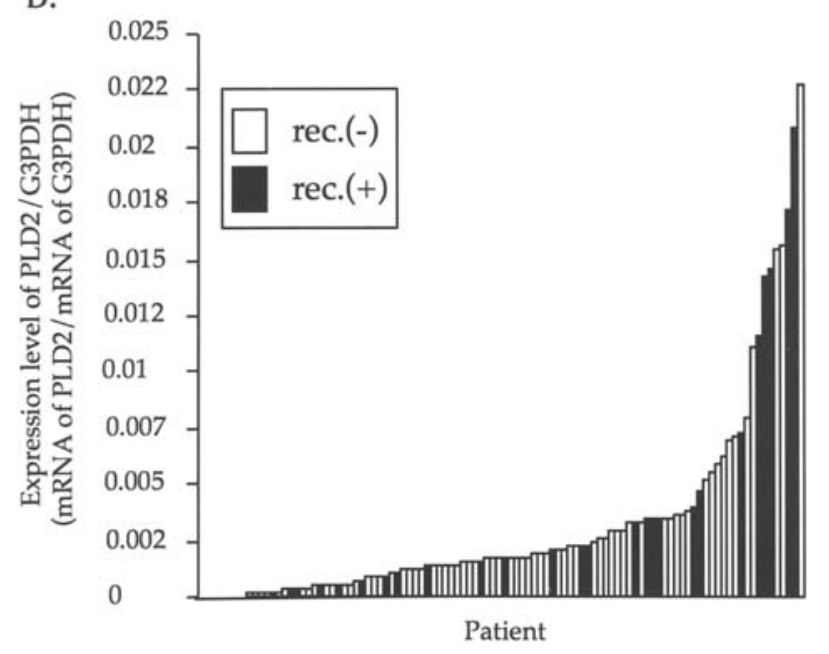

Figure 2. Expression of PLD2 in human colorectal carcinoma. (A) Expression of mRNA of PLD2 and mRNA of G3PDH in colorectal carcinoma tissues detected by RT-PCR. (B) Distribution of expression of PLD2/G3PDH (mRNA of PLD2/mRNA of G3PDH). White bars, no recurrence; black bars, recurrence of patients.

clinicopathologic features and PLD2 expression level in colorectal carcinomas. However, no significant association was found between PLD2 expression level and age, gender, 

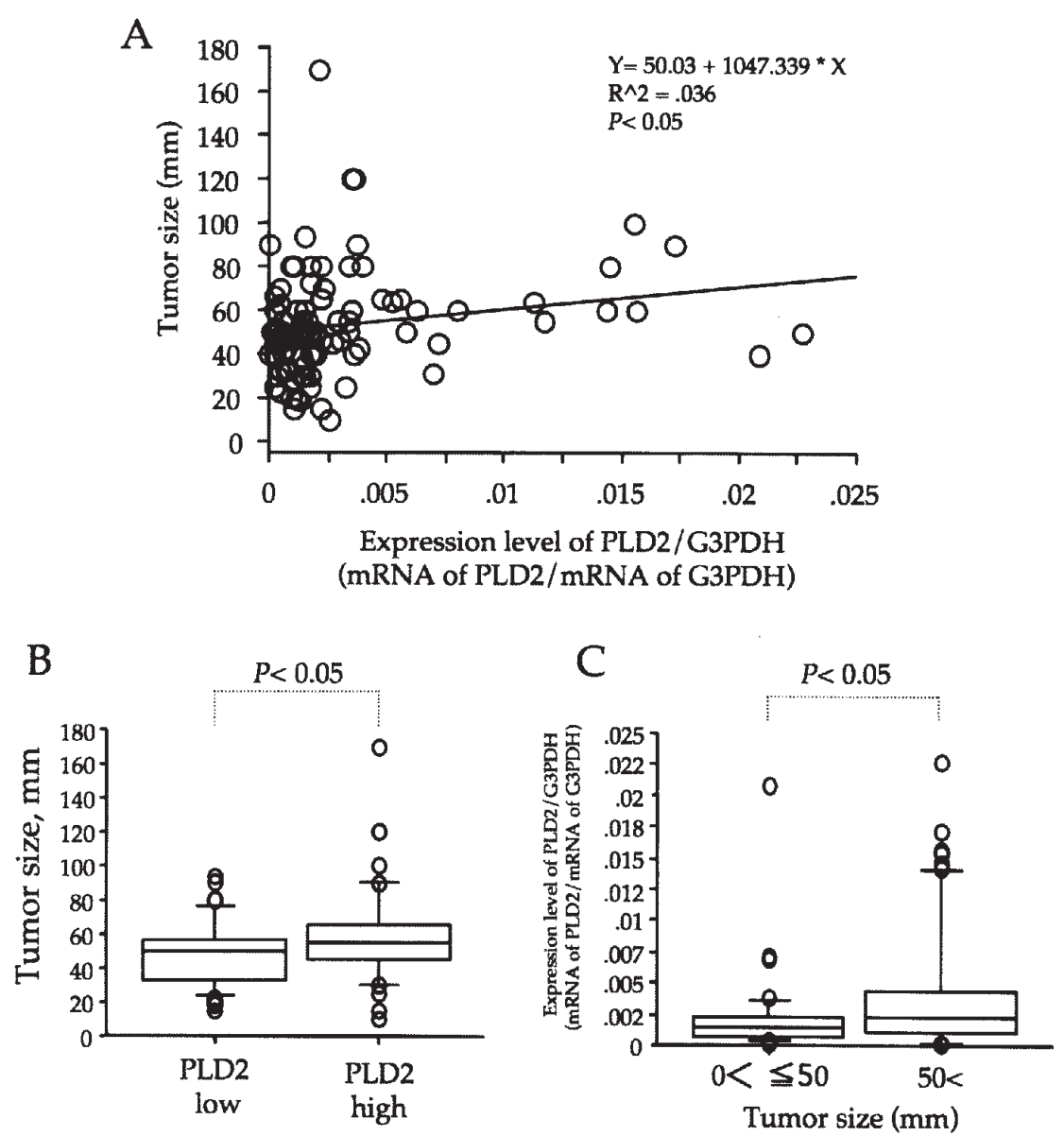

Figure 3. The relationship of tumor size and expression of PLD2 in human colorectal carcinoma. (A) Analysis of variance between tumor size and PLD2 in colorectal carcinoma. Y, tumor size; X, expression of PLD2/G3PDH (mRNA of PLD2/mRNA of G3PDH), R, regression coefficient between X and Y. Two-sided P-values were obtained by Spearman's test and linear regression analysis. (B and C) In each panel, the box corresponds to the interquartile ranges, with the lower boundary of the box representing the 25th percentile and the upper boundary representing the 75th percentile. The line inside the box represent the mean value. The vertical line represent the 5th and 95th percentiles and the open ovals present the outliers. P-values were calculated by Student's t-test (twosided).

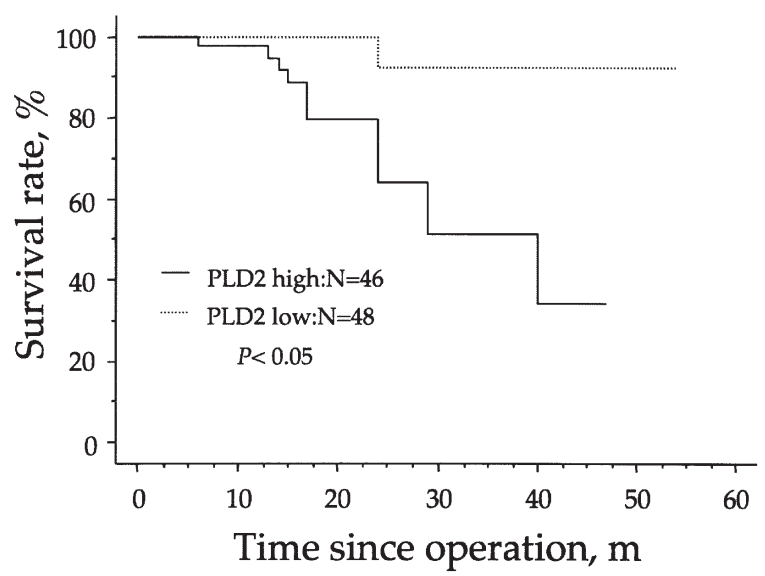

Figure 4. Kaplan-Meier survival curves of patients with colorectal carcinoma. Comparison of survival curves for patients whose tumors expressed highly PLD2 with those patients whose tumors expressed lowly PLD2. Curves present the results for all patients. Significance between two curves was conducted by log-rank test.

tumor location, histological type, extent of depth, lymph node metastasis, distant metastasis, recurrence, and Dukes' stage (Table I).
Dependency of PLD2 expression on tumor size. Fig. 3A shows the relationship between tumor size and PLD2 expression in colorectal carcinoma. PLD2 expression level was correlated with tumor size $(\mathrm{P}<0.05)$. Fig. $3 \mathrm{~B}$ shows that the average size ( \pm standard deviation) of tumor in PLD2 high expression carcinoma $(59.6 \pm 29.1 \mathrm{~mm})$ was larger than that in PLD2 low expression carcinoma $(47.7 \pm 18.6 \mathrm{~mm})$ $(\mathrm{P}<0.05)$. PLD2 expression level in large carcinoma $(>50 \mathrm{~mm})$ was significantly higher than that in small carcinoma $(\leq 50 \mathrm{~mm})(\mathrm{P}<0.05)$ (Fig. 3C).

Prognostic relevance of PLD2 expression. Kaplan-Meier estimate of overall survival are plotted in Fig. 4. The patients with PLD2 high expression carcinoma had significantly poorer survival than those with PLD2 low expression carcinoma $(\mathrm{P}<0.05)$.

\section{Discussion}

The observation that PLD2 expression and activity is elevated in several types of human solid carcinomas including breast carcinoma $(16,17)$, gastric carcinoma (18), renal carcinoma (19) and colorectal carcinoma (20) using small number of tumors, has now been confirmed and extended in 97 colorectal 
carcinoma tissues using modified real-time PCR. Herein, we demonstrated that PLD expression level is significantly correlated with tumor size (Fig. 3), and that Kaplan-Meier curve analysis reveals that the prognosis of the patients with high PLD-tumor is significantly poorer rather than that with PLD2 negative-tumor (Fig. 4).

Generally, immunohistochemical analysis of target molecules lack quantification, and results depend on techniques of laboratories, including tissue fixation, antibodies and procedures. Therefore, to observe the gene expression level more precisely and objectively, we developed the modified real-time PCR using the plasmid including each PCR product as a control for the quantification (Fig. 1). If the same Taq polymerase, cDNA produced by the same kit and the same PCR machine were used with plasmid containing PCR product as a control, similar results cloud be obtained in any laboratory. Therefore, we tried to find the clinicopathologic findings of PLD2 expression level in 97 colorectal carcinomas using modified real-time PCR in the present study.

The ability of PLD to prevent apoptosis in cells with elevated tyrosine kinase activity (21) and cooperate with tyrosine kinases to transform cells $(22,23)$ could make PLD a good candidate as oncogene in certain types of carcinomas. For example, tyrosine kinases, such as the EGF receptor, Her2/Neu, and c-Src (24), are frequently elevated in breast carcinoma. Consistent with this possibility, elevated expression of PLD and elevated PLD activity was reported in breast carcinoma tissues $(16,17)$. PLD activity has also been reported to be elevated in gastric (18) and renal carcinoma (19) and a polymorphism of the PLD2 gene was reported to be associated with the prevalence of colorectal carcinoma (25). However, the number of examined cases was $<22$ cases in all the reports described above (16-20). Therefore, we analyzed PLD2 expression level using 97 colorectal carcinoma tissues. In the present study, expression level of PLD2 varied from tumor to tumor (Fig. 2). Further, PLD2 expression levels detected by real-time PCR were significantly correlated with tumor size (Fig. 3). PI3K 1 recruits and activates Akt via phosphorylation by PDK1. Akt then phosphorylates and inactivates several substrate proteins that negatively regulate cell proliferation or stimulate apoptosis including GSK3ß, $\mathrm{BAD}$, forkhead family transcription factors $(F K H R)$, and MDM2 (26). Recently, Foster and co-workers demonstrated that an alternative survival signal that is dependent on PLD and mTOR, is active in a breast cancer cell line where the PI3K survival pathway is not active (27). Akt also activates mTOR indirectly, which indicates that there is overlap in the two pathways. The PLD/mTOR pathway involves the generation of PA, which leads to the activation of mTOR. mTOR then phosphorylates several substrate proteins that regulate protein synthesis. It has been proposed that regulators of protein synthesis provide survival signals (28). With these findings and our present data, PLD2 may contribute to the progression of colorectal carcinoma in clinic.

Although multivariate survival analysis for PLD2 expression level was not an independent prognostic factor (data not shown), the present data also suggested that PLD2 expression level detected by real-time PCR could be a prognostic indicator in human colorectal carcinoma (Fig. 4), since only the 10 cases with colorectal carcinoma have died of disease. To confirm the prognostic significance, the analysis of PLD2 expression in larger number of cases is required.

In conclusion, we demonstrated that PLD2 expression level detected by real-time PCR may be a prognostic indicator in colorectal carcinoma. Of especial interest was the finding that expression of PLD2 was dependent on tumor size. Drugs targeting PLD2 might be useful in combination with other chemotherapeutic agents for the improvement of patients with colorectal carcinoma.

\section{References}

1. Exton JH: Regulation of phospholipase D. FEBS Lett 531: 58-61, 2002.

2. Plevin R, Cook SJ, Palmer S and Wakelam MJ: Multiple sources of sn-1,2-diacylglycerol in platelet-derived-growthfactor-stimulated Swiss 3T3 fibroblasts. Evidence for activation of phosphoinositidase $\mathrm{C}$ and phosphatidylcholine-specific phospholipase D. Biochem J 279: 559-565, 1991.

3. Motoike T, Bieger S, Wiegandt $\mathrm{H}$ and Unsicker K: Induction of phosphatidic acid by fibroblast growth factor in cultured baby hamster kidney fibroblasts. FEBS Lett 332: 164-168, 1993.

4. Sa G and Das T: Basic fibroblast growth factor stimulates cytosolic phospholipase A2, phospholipase C-gammal and phospholipase D through distinguishable signaling mechanisms. Mol Cell Biochem 198: 19-30, 1999.

5. Song J, Jiang YW and Foster DA: Epidermal growth factor induces the production of biologically distinguishable diglyceride species from phosphatidylinositol and phosphatidylcholine via the independent activation of type $\mathrm{C}$ and type $\mathrm{D}$ phospholipases. Cell Growth Differ 5: 79-85, 1994.

6. Karnam P, Standaert ML, Galloway L, et al: Activation and translocation of Rho (and ADP ribosylation factor) by insulin in rat adipocytes. Apparent involvement of phosphatidylinositol 3-kinase. J Biol Chem 272: 6136-6140, 1997.

7. Banno Y, Takuwa Y, Yamada M, Takuwa N, Ohguchi K, Hara A and Nozawa Y: Involvement of phospholipase D in insulin-like growth factor-I-induced activation of extracellular signalregulated kinase, but not phosphoinositide 3-kinase or Akt, in Chinese hamster ovary cells. Biochem J 369: 363-368, 2003.

8. Zhu T, Ling L and Lobie PE: Identification of a JAK2-independent pathway regulating growth hormone $(\mathrm{GH})$-stimulated p44/42 mitogen-activated protein kinase activity. GH activation of Ral and phospholipase D is Src-dependent. J Biol Chem 277: 45592-45603, 2002.

9. Banno Y, Takuwa Y, Akao Y, Okamoto H, Osawa Y, Naganawa T, Nakashima S, Suh PG and Nozawa Y: Involvement of phospholipase D in sphingosine 1-phosphate-induced activation of phosphatidylinositol 3-kinase and Akt in Chinese hamster ovary cells overexpressing EDG3. J Biol Chem 276: 35622-35628, 2001

10. Song JG, Pfeffer LM and Foster DA: v-Src increases diacylglycerol levels via a type D phospholipase-mediated hydrolysis of phosphatidylcholine. Mol Cell Biol 11: 4903-4908, 1991.

11. Carnero A, Cuadrado A, Del Peso L and Lacal JC: Activation of type D phospholipase by serum stimulation and ras-induced transformation in NIH3T3 cells. Oncogene 9: 1387-1395, 1994.

12. Jiang YW, Song J, Zang Q and Foster DA: Phosphatidylcholine-specific phospholipase D activity is elevated in v-Fpstransformed cells. Biochem Biophys Res Commun 203: 11952003, 1994 .

13. Frankel P, Ramos M, Flom J, Bychenok S, Joseph T, Kerkhoff E, Rapp UR, Feig LA and Foster DA: Ral and Rho-dependent activation of phospholipase D in v-Raf-transformed cells. Biochem Biophys Res Commun 255: 502-507, 1999.

14. Welsh CJ, Yeh GC and Phang JM: Increased phospholipase D activity in multidrug resistant breast cancer cells. Biochem Biophys Res Commun 202: 211-217, 1994.

15. Chen Y, Zheng Y and Foster DA: Phospholipase D confers rapamycin resistance in human breast cancer cells. Oncogene 22: 3937-3942, 2003.

16. Uchida N, Okamura S, Nagamachi Y and Yamashita S: Increased phospholipase D activity in human breast cancer. J Cancer Res Clin Oncol 123: 280-285, 1997. 
17. Noh DY, Ahn SJ, Lee RA, Park IA, Kim JH, Suh PG, Ryu SH, Lee KH and Han JS: Overexpression of phospholipase D1 in human breast cancer tissues. Cancer Lett 161: 207-214, 2000.

18. Uchida, N, Okamura, S and Kuwano H: Phospholipase D activity in human gastric carcinoma. Anticancer Res 19: 671-675, 1999.

19. Zhao Y, Ehara H, Akao Y, Shamoto M, Nakagawa Y, Banno Y, Deguchi T, Ohishi N, Yagi K and Nozawa Y: Increased activity and intranuclear expression of phospholipase D2 in human renal cancer. Biochem Biophys Res Commun 278: 140-143, 2000.

20. Oshimoto H, Okamura S, Yoshida M and Mori M: Increased activity and expression of phospholipase D2 in human colorectal cancer. Oncol Res 14: 31-37, 2003.

21. Zhong M, Shen Y, Zheng Y, Joseph T, Jackson D and Foster DA: Phospholipase D prevents apoptosis in $\mathrm{v}$-Src-transformed rat fibroblasts and MDA-MB-231 breast cancer cells. Biochem Biophys Res Commun 302: 615-619, 2003.

22. Lu Z, Hornia A and Joseph T: Phospholipase D and RalA cooperate with the epidermal growth factor receptor to transform 3 Y1 rat fibroblasts. Mol Cell Biol 20: 462-467, 2000.
23. Joseph T, Wooden R, Bryant A, Zhong M, Lu Z and Foster DA Transformation of cells overexpressing a tyrosine kinase by phospholipase D1 and D2. Biochem Biophys Res Commun 289: 1019-1024, 2001.

24. Biscardi JS, Tice DA and Parsons SJ: c-Src, receptor tyrosine kinases, and human cancer. Adv Cancer Res 76: 61-119, 1999.

25. Yamada Y, Hamajima N, Kato T, Iwata H, Yamamura Y, Shinoda M, Suyama M, Mitsudomi T, Tajima K, Kusakabe S, Yoshida H, Banno Y, Akao Y, Tanaka M and Nozawa Y: Association of a polymorphism of the phospholipase D2 gene with the prevalence of colorectal cancer. J Mol Med 81: 126-131, 2003.

26. Vivanco I and Sawyers CL: The phosphatidylinositol 3-Kinase AKT pathway in human cancer. Nat Rev Cancer 2: 489-501, 2002.

27. Chen Y, Rodrik V and Foster DA: Alternative phospholipase $\mathrm{D} / \mathrm{mTOR}$ survival signal in human breast cancer cells. Oncogene 24: 672-679, 2005.

28. Ruggero D and Pandolfi PP: Does the ribosome translate cancer? Nat Rev Cancer 3: 179-192, 2003. 